\title{
Confinement from gluodynamics in curved space-time
}

\author{
Patricio Gaete* \\ Departamento de Física, Universidad Técnica Federico Santa María, Valparaíso, Chile \\ Euro Spallucc $\oplus$ \\ Dipartimento di Fisica Teorica, Università di Trieste and INFN, Sezione di Trieste, Italy
}

(Dated: October 30, 2018)

\begin{abstract}
We determine the static potential for a heavy quark-antiquark pair from gluodynamics in curved space-time. Our calculation is done within the framework of the gauge-invariant, path-dependent, variables formalism. The potential energy is the sum of a Yukawa and a linear potential, leading to the confinement of static charges.

PACS numbers: 11.10.Ef, 11.15.-q
\end{abstract}

\section{INTRODUCTION}

One of the fundamental issues facing QCD at low energy is a quantitative description of confinement. We observe at this point that the distinction between the apparently related phenomena of screening and confinement is of considerable importance in our present understanding of gauge theories. In fact, gauge theories that yield a linear potential are important to particle physics, since those theories may be used to describe the confinement of quarks and gluons and be considered as effective theories of QCD. As is well known, the confinement problem has been fairly well discussed under a number different aspects, like lattice gauge theory techniques [1] and non-perturbative solutions of Schwinger-Dyson's equations 2, 3]. Recently, an appealing proposal to this problem was made by 't Hooft [4] which includes a linear term in the dielectric field that appears in the energy density.

In this connection it becomes of interest, in particular, to recall that QCD at the classical level possesses scale invariance which is broken by quantum effects. We further observe that this phenomenon can be mathematically described by formulating classical gluodynamics in a curved space-time with non vanishing cosmological constant [5, 6]. Correspondingly, an effective low energy Lagrangian for gluodynamics which describes semi-classical vacuum fluctuations of gluon field at large distances is obtained [5, 6].

In the light of the above observations, we also mention that the Cornell potential 7] which simulates the features of $Q C D$ is given by

$$
V=-\frac{\kappa}{r}+\frac{r}{a^{2}}
$$

where $a$ is a constant with the dimensions of length. In accordance with the 't Hooft proposal, confinement is

\footnotetext{
*Electronic address: patricio.gaete@usm.cl

${ }^{\dagger}$ Electronic address: euro@ts.infn.it
}

associated to the appearance of a linear term in the dielectric field $\mathbf{D}$ (that dominates for low $|\mathbf{D}|$ ) in the energy density [4]:

$$
U(\mathbf{D})=\rho_{s t r}|\mathbf{D}|
$$

the proportionality constant being the coefficient of the linear potential, that is, $\rho_{s t r} \approx \frac{1}{a^{2}}$. Hence we see that the confinement phenomena breaks the scale invariance as the Cornell potential explicitly shows by introducing the scale $a$.

Very recently [8, 9], we have approached the connection between scale symmetry breaking and confinement in a phenomenological way using the gauge-invariant but path-dependent variables formalism, which is alternative to the Wilson loop approach. More specifically, we have shown the appearance of the Cornell potential (11) as well as the 't Hooft relation (2) after spontaneous breaking of scale invariance in both Abelian and non-Abelian cases. Certainly, this study gives us an opportunity to compare our results with that of gluodynamics in curved spacetime $[\underline{5}, 6]$. To this end, we will work out the static potential for the theory under consideration along the lines of Ref. [8, 9]. As a result, it is found that the potential energy is the sum of a Yukawa and a linear potential, leading to the confinement of static charges. This static potential clearly shows the key role played by the quantum fluctuations. In general, this picture agrees qualitatively with that encountered in our previous phenomenological model [9]. It is important to realize that the gluodynamics in curved space-time studied here, compared with our previous model which includes a $\sqrt{F_{\mu \nu}^{a} F^{a \mu \nu}}$ term coupled to the Yang-Mills Lagrangian density, involves the contribution of quantum fluctuations. Hence we see that our phenomenological model incorporates automatically the contribution of these quantum fluctuations to the vacuum of the model. In this way we establish a new correspondence between these two non-Abelian effective theories. The above connections are of interest from the point of view of providing unifications among diverse models as well as exploiting the equivalence in explicit calculations. 


\section{INTERACTION ENERGY}

We now examine the interaction energy between static point-like sources for gluodynamics in curved space-time. To do this, we will compute the expectation value of the energy operator $H$ in the physical state $|\Phi\rangle$ describing the sources, which we will denote by $\langle H\rangle_{\Phi}$. However, before going to the derivation of the interaction energy, we will describe very briefly the model under consideration. The initial point of our analysis is the dilaton effective lagrangian coupled to gluodynamics [5]:

$$
\begin{aligned}
\mathcal{L} & =\frac{\left|\varepsilon_{V}\right|}{m^{2}} \frac{1}{2} e^{\chi / 2}\left(\partial_{\mu} \chi\right)^{2}+\left|\varepsilon_{V}\right| e^{\chi}(1-\chi) \\
& -e^{\chi}(1-\chi) \frac{1}{4} F_{\mu \nu}^{a} F^{a \mu \nu},
\end{aligned}
$$

where the real scalar field $\chi$ of mass $m$ represents the dilaton, and $-\left|\varepsilon_{V}\right|$ is the vacuum energy density.

By expanding near the $\chi=0$, expression (3) then becomes

$$
\begin{aligned}
\mathcal{L} & =-\frac{1}{4} F_{\mu \nu}^{a} F^{a \mu \nu}+\frac{\left|\varepsilon_{V}\right|}{2 m^{2}}\left(\partial_{\mu} \chi\right)^{2} \\
& -\chi\left(\left|\varepsilon_{V}\right|-\frac{1}{4}\left(F_{\mu \nu}^{a}\right)^{2}\right)
\end{aligned}
$$

Following our earlier procedure [8, 9], integrating out the $\chi$ field induces an effective theory for the $A_{\mu}^{a}$ field. Once this is done, we arrive at the following effective Lagrangian density:

$$
\begin{aligned}
\mathcal{L}_{e f f} & =-\frac{1}{4} F_{\mu \nu}^{a}\left(1+\frac{m^{2}}{\Delta}\right) F^{a \mu \nu} \\
& +\frac{m^{2}}{32\left|\varepsilon_{V}\right|}\left(F_{\mu \nu}^{a}\right)^{2} \frac{1}{\Delta}\left(F_{\mu \nu}^{a}\right)^{2} .
\end{aligned}
$$

Next, in order to linearize this theory, we introduce the auxiliary field $\phi$. It follows that the expression (5) can be rewritten as

$$
\begin{aligned}
\mathcal{L}_{e f f} & =-\frac{1}{4} F_{\mu \nu}^{a}\left(1+\frac{m^{2}}{\Delta}\right) F^{a \mu \nu}+\frac{1}{2}\left(\partial_{\mu} \phi\right)^{2} \\
& -\frac{1}{4} \frac{m}{\sqrt{\left|\varepsilon_{V}\right|}} \phi\left(F_{\mu \nu}^{a}\right)^{2} .
\end{aligned}
$$

Once again, by expanding about $\phi=\phi_{0}$, we then obtain

$$
\mathcal{L}_{e f f}=-\frac{1}{4} F_{\mu \nu}^{a} \frac{1}{\varepsilon}\left(1+\frac{\varepsilon m^{2}}{\Delta}\right) F^{a \mu \nu},
$$

where $\frac{1}{\varepsilon} \equiv 1+\frac{m}{\sqrt{\left|\varepsilon_{V}\right|}} \phi_{0}$.

To obtain the corresponding Hamiltonian, we must carry out the quantization of this theory. The Hamiltonian analysis starts with the computation of the canonical momenta $\Pi^{a \mu}=-\frac{1}{\varepsilon}\left(1+\frac{\varepsilon m^{2}}{\Delta}\right) F^{a 0 \mu}$, and one immediately identifies the primary constraint $\Pi^{a 0}=0$ and
$\Pi^{a i}=-\frac{1}{\varepsilon}\left(1+\frac{\varepsilon m^{2}}{\Delta}\right) F^{a 0 i}$. Standard techniques for constrained systems then lead to the following canonical Hamiltonian:

$$
\begin{aligned}
H_{C} & =\int d^{3} x\left\{\frac{\varepsilon}{2} \Pi^{a i}\left(1+\frac{\varepsilon m^{2}}{\Delta}\right)^{-1} \Pi^{a i}\right\} \\
& +\int d^{3} x\left\{\frac{1}{4 \varepsilon} F_{i j}^{a}\left(1+\frac{\varepsilon m^{2}}{\Delta}\right) F^{a i j}\right\} \\
& +\int d^{3} x\left\{\Pi^{a i}\left(\partial_{i} A_{0}^{a}+g f^{a b c} A_{0}^{c} A_{i}^{b}\right)\right\} .
\end{aligned}
$$

The persistence of the primary constraints $\Pi^{a 0} \approx 0$ leads to the following secondary constraints $\Gamma^{a(1)}(x) \equiv$ $\partial_{i} \Pi^{a i}+g f^{a b c} A^{b i} \Pi_{i}^{c} \approx 0$. It is easy to check that there are no further constraints, and that the above constraints are first class. Therefore, the extended Hamiltonian that generates translations in time then reads $H=H_{C}+\int d x\left(c_{0}^{a}(x) \Pi_{0}^{a}(x)+c_{1}^{a}(x) \Gamma^{a(1)}(x)\right)$, where $c_{0}^{a}(x)$ and $c_{1}^{a}(x)$ are the Lagrange multipliers. Moreover, it follows from this Hamiltonian that $\dot{A}_{0}^{a}(x)=$ $\left[A_{0}^{a}(x), H\right]=c_{0}^{a}(x)$, which are arbitrary functions. Since $\Pi^{0 a}=0$, neither $A^{0 a}$ nor $\Pi^{0 a}$ are of interest in describing the system and may be discarded from the theory. The Hamiltonian then takes the form

$$
\begin{aligned}
H & =\int d^{3} x\left\{\frac{\varepsilon}{2} \Pi^{a}\left(1+\frac{\varepsilon m^{2}}{\Delta}\right)^{-1} \boldsymbol{\Pi}^{a}\right\} \\
& +\int d^{3} x\left\{\frac{1}{2 \varepsilon} \mathbf{B}^{a}\left(1+\frac{\varepsilon m^{2}}{\Delta}\right) \mathbf{B}^{a}\right\} \\
& +\int d^{3} x\left\{c^{a}(x)\left(\partial_{i} \Pi^{a i}+g f^{a b c} A^{b i} \Pi_{i}^{c}\right)\right\} .
\end{aligned}
$$

where $c^{a}(x)=c_{1}^{a}(x)-A_{0}^{a}(x)$. According to the usual procedure we introduce a supplementary condition on the vector potential such that the full set of constraints becomes second class. A particularly useful and interesting choice is given by [10]:

$$
\Gamma^{a(2)}(x)=\int_{0}^{1} d \lambda(x-\xi)^{i} A_{i}^{(a)}(\xi+\lambda(x-\xi)) \approx 0,
$$

where $\lambda(0 \leq \lambda \leq 1)$ is the parameter describing the spacelike straight path $x^{i}=\xi^{i}+\lambda(x-\xi)^{i}$, on a fixed time slice. Here $\xi$ is a fixed point (reference point), and there is no essential loss of generality if we restrict our considerations to $\xi^{i}=0$. As a consequence, the only nontrivial Dirac bracket is

$$
\begin{array}{r}
\left\{A_{i}^{a}(x), \Pi^{b j}(y)\right\}^{*}=\delta^{a b} \delta_{i}^{j} \delta^{(3)}(x-y) \\
-\int_{0}^{1} d \lambda\left(\delta^{a b} \frac{\partial}{\partial x^{i}}-g f^{a b c} A_{i}^{c}(x)\right) x^{j} \delta^{(3)}(\lambda x-y) .
\end{array}
$$

In passing we note the presence of the last term on the right-hand side which depends on $g$. 
Now we move on to compute the interaction energy between pointlike sources in the theory under consideration, where a fermion is localized at the origin $\mathbf{0}$ and an antifermion at $\mathbf{y}$. As mentioned before, in order to accomplish this purpose we will calculate the expectation value of the energy operator $H$ in the physical state $|\Phi\rangle$. From our above discussion we see that $\langle H\rangle_{\Phi}$ reads

$$
\langle H\rangle_{\Phi}=\frac{\varepsilon}{2} \operatorname{tr}\left\langle\Phi\left|\int d^{3} x \Pi^{a i}\left(1+\frac{\varepsilon m^{2}}{\Delta}\right)^{-1} \Pi^{a i}\right| \Phi\right\rangle .
$$

At this stage we recall that the physical state can be written as [10],

$$
|\Phi\rangle=\bar{\psi}(\mathbf{y}) U(\mathbf{y}, \mathbf{0}) \psi(\mathbf{0})|0\rangle
$$

where

$$
U(\mathbf{y}, \mathbf{0}) \equiv P \exp \left(i g \int_{\mathbf{0}}^{\mathbf{y}} d z^{i} A_{i}^{a}(z) T^{a}\right) .
$$

As before, the line integral is along a spacelike path on a fixed time slice, $P$ is the path-ordering prescription and $|0\rangle$ is the physical vacuum state. As in [10], we again restrict our attention to the weak coupling limit.

From the above Hamiltonian analysis the static potential is divided into two parts: an Abelian part $V^{(1)}$ (proportional to $C_{F}$ ) and a non-Abelian part $V^{(2)}$ (proportional to the combination $C_{F} C_{A}$ ). Thus $\langle H\rangle_{\Phi}$ takes the form

$$
\langle H\rangle_{\Phi}=\langle H\rangle_{0}+V^{(1)}+V^{(2)}
$$

where $\langle H\rangle_{0}=\langle 0|H| 0\rangle$. The $V^{(1)}$ and $V^{(2)}$ terms are given by

$$
\begin{aligned}
V^{(1)} & =-\frac{g^{2}}{2} \varepsilon \operatorname{tr}\left(T^{a} T^{a}\right) \int d^{3} x \int_{\mathbf{0}}^{\mathbf{y}} d z_{i}^{\prime} \delta^{(3)}\left(\mathbf{x}-\mathbf{z}^{\prime}\right) \\
& \times \frac{1}{\nabla_{x}^{2}-\varepsilon m^{2}} \nabla_{x}^{2} \int_{\mathbf{0}}^{\mathbf{y}} d z^{i} \delta^{(3)}(\mathbf{x}-\mathbf{z})
\end{aligned}
$$

and

$$
\begin{aligned}
V^{(2)} & =\frac{\varepsilon}{2} \mathcal{N}\langle 0| \int_{\mathbf{0}}^{\mathbf{y}} d z^{\prime k} A_{k}^{c}\left(z^{\prime}\right) z^{i} \int_{0}^{1} d \lambda \delta^{(3)}\left(\lambda \mathbf{z}^{\prime}-\mathbf{x}\right) \\
& \times \frac{1}{\nabla_{x}^{2}-\varepsilon m^{2}} \nabla_{x}^{2} \int_{\mathbf{0}}^{\mathbf{y}} d z^{l} A_{l}^{e}(z) \\
& \times z^{i} \int_{0}^{1} d \beta \delta^{(3)}(\beta \mathbf{z}-\mathbf{x})|0\rangle
\end{aligned}
$$

where $\mathcal{N}=g^{4} \operatorname{tr}\left(f^{a b c} T^{b} f^{a d e} T^{d}\right)$ and the integrals over $z^{i}$ and $z_{i}^{\prime}$ are zero except on the contour of integration.

Here, the $V^{(1)}$ term gives a Yukawa-type potential plus self-energy terms. In effect, expression (16) can also be written as

$$
V^{(1)}=\frac{g^{2}}{2} \varepsilon \operatorname{tr}\left(T^{a} T^{a}\right) \int_{\mathbf{0}}^{\mathbf{y}} d z_{i}^{\prime} \partial_{i}^{z^{\prime}} \int_{\mathbf{0}}^{\mathbf{y}} d z^{i} \partial_{z}^{i} G\left(\mathbf{z}, \mathbf{z}^{\prime}\right),
$$

where $G$ is the Green function

$$
G\left(\mathbf{z}, \mathbf{z}^{\prime}\right)=\frac{1}{4 \pi} \frac{e^{-m \sqrt{\varepsilon}\left|\mathbf{z}-\mathbf{z}^{\prime}\right|}}{\left|\mathbf{z}-\mathbf{z}^{\prime}\right|} .
$$

Employing Eq.(19) and remembering that the integrals over $z^{i}$ and $z_{i}^{\prime}$ are zero on the contour of integration, expression (18) reduces to the familiar Yukawa potential after subtracting the self-energy terms. In other words,

$$
V^{(1)}=-\frac{g^{2}}{4 \pi} \varepsilon C_{F} \frac{e^{-m \sqrt{\varepsilon} L}}{L},
$$

where $|\mathbf{y}| \equiv L$ and $\operatorname{tr}\left(T^{a} T^{a}\right)=C_{F}$. We also recall that $\varepsilon \equiv\left(1+\frac{m}{\sqrt{\left|\varepsilon_{V}\right|}} \phi_{0}\right)^{-1}$.

We now turn our attention to the calculation of the $V^{(2)}$ term, which is given by

$$
\begin{aligned}
V^{(2)} & =\frac{\varepsilon}{2} \mathcal{N}^{\prime} \int_{\mathbf{0}}^{\mathbf{y}} d z^{l} \int_{\mathbf{0}}^{\mathbf{y}} d z^{\prime k} D_{l k}\left(\mathbf{z}, \mathbf{z}^{\prime}\right) \\
& \times \int_{\mathbf{0}}^{\mathbf{z}^{\prime}} d u^{i} \int_{\mathbf{0}}^{\mathbf{z}} d v^{i}\left(-\nabla^{2}\right)_{\mathbf{u}} G(\mathbf{u}, \mathbf{v}),
\end{aligned}
$$

where $\mathcal{N}^{\prime}=g^{4} \operatorname{tr}\left(f^{a b c} T^{b} f^{a d c} T^{d}\right)$, and $G$ is the Green function. Here $D_{l k}\left(\mathbf{z}, \mathbf{z}^{\prime}\right)$ stands for the propagator, which is diagonal in color space and taken in an arbitrary gauge.

It is appropriate to observe here that the above term is similar to the one found in non-Abelian axionic electrodynamics [11]. Nevertheless, in order to put our discussion into context it is useful to summarize the relevant aspects of the analysis described previously [11]. In effect, as was observed in Ref. [11], by writing the Green function in momentum space

$$
G(\mathbf{u}, \mathbf{v})=\int \frac{d^{3} k}{(2 \pi)^{3}} \frac{e^{i \mathbf{k} \cdot(\mathbf{u}-\mathbf{v})}}{\mathbf{k}^{2}+\varepsilon m^{2}}
$$

expression (21) reduces to

$$
\begin{aligned}
V^{(2)} & =\frac{\varepsilon}{2} \mathcal{N}^{\prime} \int_{\mathbf{0}}^{\mathbf{y}} d z^{l} \int_{\mathbf{0}}^{\mathbf{y}} d z^{\prime k} D_{l k}\left(\mathbf{z}, \mathbf{z}^{\prime}\right) \\
& \times \int_{\mathbf{0}}^{\mathbf{z}^{\prime}} d u^{i} \int_{\mathbf{0}}^{\mathbf{z}} d v^{i} \int \frac{d^{3} k}{(2 \pi)^{3}} \mathbf{k}^{2} \frac{e^{i \mathbf{k} \cdot(\mathbf{u}-\mathbf{v})}}{\mathbf{k}^{2}+\varepsilon m^{2}}
\end{aligned}
$$

Following our earlier procedure [11], equation (23) can also be written as

$$
V^{(2)}=\frac{g^{4}}{8 \pi} \varepsilon C_{F} C_{A} \sigma \int_{\mathbf{0}}^{\mathbf{y}} d z^{l} \int_{\mathbf{0}}^{\mathbf{y}} d z^{\prime k}|\mathbf{z}| D_{l k}\left(\mathbf{z}, \mathbf{z}^{\prime}\right),
$$

where $\sigma \equiv\left[\Lambda^{2}-\varepsilon m^{2} \ln \left(1+\frac{\Lambda^{2}}{\varepsilon m^{2}}\right)\right]$, and $\Lambda$ is a cutoff. As has been shown previously [11], we choose $D_{l k}\left(\mathbf{z}, \mathbf{z}^{\prime}\right)$ in the Feynman gauge. As a consequence, expression (24) then becomes

$$
V^{(2)}=\frac{g^{4}}{8 \pi} \varepsilon C_{F} C_{A} \sigma L .
$$


From equations (20) and (25), the corresponding static potential for two opposite charges located at $\mathbf{0}$ and $\mathbf{y}$ may be written as

$$
V=-\frac{g^{2}}{4 \pi} \varepsilon C_{F} \frac{e^{-m \sqrt{\varepsilon} L}}{L}+\frac{g^{4}}{8 \pi} \varepsilon C_{F} C_{A} \sigma L,
$$

where $|\mathbf{y}| \equiv L$, and $\varepsilon \equiv\left(1+\frac{m}{\sqrt{\left|\varepsilon_{V}\right|}} \phi_{0}\right)^{-1}$. This potential displays the conventional screening part, encoded in the Yukawa potential, and the linear confining potential. It is worthwhile noticing that the result (26) leads to a Coulomb-type potential in the limit of large $m$. This then implies that expression (26) has the Cornell form.

\section{FINAL REMARKS}

We have studied the equivalence between two nonAbelian effective theories. To this end we have computed the static potential for a QCD effective theory which represents propagation and interaction of gluons and dilaton. As a consequence of this the potential energy is the sum of a Yukawa and a linear potential, leading to the confinement of static charges. In a general perspective, this picture agrees qualitatively with that encountered in our previous phenomenological model [9]. We also mention that this result is in agreement with the studies of Ref. [12].

In this way we have provided a new connection between effective models. The above analysis reveals the key role played by the quantum fluctuations in order to obtain confinement.

\section{ACKNOWLEDGMENTS}

Work supported in part by FONDECYT (Chile) grant 1050546 .

[1] K. G. Wilson, Phys. Rev. D10, 2445 (1974).

[2] M. Baker, J. S. Ball and F. Zachariasen, Phys. Rept. 209, 73 (1991).

[3] D. Epple, H. Reinhardt and W. Schleifenbaum, Phys. Rev. D75, 045011 (2007).

[4] G. 't Hooft, Nucl. Phys. Proc. Suppl. 121, 333 (2003).

[5] D. Kharzeev, E. Levin and K. Tuchin, Phys. Lett. B547, 21 (2002).

[6] D. Kharzeev, E. Levin and K. Tuchin, Phys. Rev. D70, 054005 (2004).

[7] E. Eichten, K. Gottfried, T. Kinoshita, K. D. Lane, and
T. M. Yan, Phys. Rev. D17, 3090 (1978).

[8] P. Gaete and E. Guendelman, Phys. Lett. B640, 201 (2006).

[9] P. Gaete, E. Guendelman and E. Spallucci, Phys. Lett. B649, 218 (2007).

[10] P. Gaete, Phys. Lett. B515, 382 (2001).

[11] P. Gaete and E. Spallucci, J. Phys. A39, 6021 (2006).

[12] R. Dick, Phys. Lett. B397, 193 (1997); Phys. Lett. B409, 321 (1997). 